\title{
Current Decoupling and Controller Design for Induction Motors Based on Neutral Delay System
}

\author{
Guofang Wang ${ }^{1}$, Yuedou $\mathrm{Pan}^{1}$, and Yongliang $\mathrm{Li}^{1}$ \\ ${ }^{1}$ School of Automation, University of Science and Technology Beijing, Beijing, 100083, China
}

\begin{abstract}
In the AC power transmission system, in order to reduce the loss of switching devices, the switching frequency of the traction converter is generally low, and a large digital control delay will occur, which will aggravate the cross coupling between the excitation component and the torque component of the stator current of the motor, resulting in poor system performance. In order to solve this problem, based on the theory of neutral time-delay system and the rotor flux-oriented model of induction motors, the mathematical model of neutral-type time-delay system for induction motors is established, and a neutral current controller with current decoupling control is designed. The decoupling control of the stator current reduces the influence of the digital control delay on the system performance. The simulation results show that the induction motor system with a neutral current controller has the advantages of small coupling, rapid response, and strong robustness. Explains the feasibility of the designed current controller.
\end{abstract}

\section{Introduction}

In engineering applications, time lags are ubiquitous, and time lags can bring about certain consequences, ranging from poor system performance to system oscillations [1]. Delay is the inherent problem of digital control. Inevitably there is a delay problem in current loop digital control systems of induction motors. Especially in AC high-power drive systems, the switching frequency of traction converters is generally low in order to reduce switching device losses. It will produce a large digital control delay, aggravating the cross-coupling of the motor excitation component and torque component of stator current [2]. Current loop implementation methods are based on the premise of small delay and coupling, and do not solve the problem of poor control performance of the drive system when the switching frequency is low.

K. K. Wei and M. L. Zhou [3] proposed a digital control method based on complex vector with delay compensation to improve the performance of the current loop. E. Monmasson and M. N. Cirstea [4] used high-performance programmable logic devices (FPGA) to minimize the control time, to have the characteristics of the analog domain, and to eliminate the control delay. G. H. Bode and P. C. Loh [5] used advanced control algorithms such as deadbeat and prediction to compensate for the loop delay. The basic idea of these methods is to compensate for the delay. The delay problem is not introduced into the mathematical model and the delay cannot be fundamentally solved.

In order to solve the above problems, this paper proposes a method of implementing a current controller with small coupling, fast response and strong robustness, so as to achieve a rapid, accurate, and stable control target of motor torque response.

\section{Neutral mathematical model of induction motors}

\subsection{Neutral system}

Neutral time-delay systems are a more general type of lag system. The full expression of a neutral time-delay system is as follow [6].

$$
\left\{\begin{array}{l}
\dot{x}(t)-G \dot{x}(t-\tau)=A x(t)+A_{1} x(t-\tau)+f(x(t), x(t-\tau))+B u(t) \\
x(t)=\phi(t), \quad t \in[-\tau, 0]
\end{array}\right.
$$

Where $x(t) \in R^{n}$ is the system state vector. $\tau>0$ is time delay. $A_{0}, A_{1}, G$ and $B$ are constant matrices of dimension, $\|G\|<1 . \phi(t)$ is a vector initial value function, $\phi(t) \in C\left([-\tau, 0], R^{n}\right) . \quad f(x(t), x(t-\tau))$ is an unobservable uncertain nonlinear disturbance, and satisfies the following formula.

$$
\|f(x(t), x(t-\tau))\| \leq \beta\|x\|+\alpha\|x(t-\tau)\|
$$

Where $\alpha, \beta$ is a known constant.

\subsection{State equation of the rotor flux-oriented}

In this paper, the voltage variable is taken as the input, the electromagnetic torque and the motor speed are output to establish the control model of the induction motor, and the state equation of the induction motor in the coordinate system is obtained [7]: 


$$
\left\{\begin{array}{l}
\frac{\mathrm{d} \psi_{r}}{\mathrm{dt}}=-\frac{1}{T_{r}} \psi_{r}+\frac{L_{m d}}{T_{r}} i_{s M} \\
\frac{\mathrm{d} i_{s M}}{\mathrm{dt}}=\frac{L_{m d}}{\sigma L_{s d} L_{r d} T_{r}} \psi_{r}-\frac{R_{s} L_{r d}^{2}+R_{r} L_{m d}^{2}}{\sigma L_{s d} L_{r d}^{2}} i_{s M}+\omega_{s} i_{s T}+\frac{1}{\sigma L_{s d}} u_{s M} \\
\frac{\mathrm{d} i_{s T}}{\mathrm{dt}}=-\frac{L_{m d}}{\sigma L_{s d} L_{r d}} \omega \psi_{r}-\frac{R_{s} L_{r d}^{2}+R_{r} L_{m d}^{2}}{\sigma L_{s d} L_{r d}^{2}} i_{s T}-\omega_{s} i_{s M}+\frac{1}{\sigma L_{s d}} u_{s T}
\end{array}\right.
$$

In the formula, $u_{s M}$ and $u_{s T}$ are the $\mathrm{M}$ axis components and the $\mathrm{T}$ axis components of the stator voltage under the M-T coordinate system respectively. $i_{s M}$ and $i_{s T}$ are the $\mathrm{M}$ axis components and $\mathrm{T}$ axis components of the stator current respectively. $R_{s}$ is stator winding resistance; $R_{r}$ is rotor winding resistance; $L_{m d}$ is the stator and rotor coaxial equivalent winding mutual inductance under M-T coordinate system; $L_{s d}$ and $L_{r d}$ are the equivalent two phase winding of stator and rotor in M-T coordinate system, respectively. $\psi_{r}$ is the total flux linkage vector of the rotor; $J$ is the inertia moment of the motor; $\sigma$ is the leakage flux coefficient of the motor, $\sigma=1-L_{m d}^{2} /\left(L_{s d} L_{r d}\right) ; T_{r}$ is the rotor electromagnetic time constant, $T_{r}=L_{r d} / R_{r}$.

$$
\left\{\begin{array}{l}
\frac{\mathrm{d} \omega}{\mathrm{d} t}=\frac{n_{p}^{2} L_{m d}}{J L_{r d}} i_{s T} \psi_{r}-\frac{n_{p}}{J} T_{L} \\
T_{e}=\frac{n_{p} L_{m d}}{L_{r d}} i_{s T} \psi_{r} \\
\omega_{s}=\omega+\frac{L_{m d}}{T_{r} \psi_{r}} i_{s T}(t)
\end{array}\right.
$$

Where $n_{p}$ is the extreme logarithm; $T_{L}$ is the resistance torque of the load; $T_{e}$ is the electromagnetic torque; $\omega$ is rotor rotation angular velocity; $\omega_{s}$ is synchronous angular velocity.

\subsection{Neutral delay system model of induction motors}

This paper takes into account the phenomenon of asynchronous switching of the closed-loop control of the motor and creatively proposes a neutral system for induction motors. Since the neutral model considers not only the current state but also the hysteresis state and the state differential delay term. The description of the system is more accurate.

$X=\left[\begin{array}{lll}\psi_{r} & i_{S M} & i_{s T}\end{array}\right]$ is chosen as the state variable of the system, and its equation of state is as follows:

$$
\left(\begin{array}{c}
\frac{\mathrm{d} \psi_{r}(t)}{\mathrm{dt}} \\
\frac{\mathrm{d} i_{s h}(t)}{\mathrm{dt}} \\
\frac{\mathrm{d}_{s T}(t)}{\mathrm{dt}}
\end{array}\right)=\left[\begin{array}{ccc}
-\frac{1}{T_{r}} & \frac{L_{n d}}{T_{r}} & 0 \\
\frac{L_{n d}}{\sigma L_{s d} L_{n d} T_{r}} & -\frac{R L_{n d}^{2}+R L_{r d}^{2}}{\sigma L_{s d} L_{n d}^{2}} & \omega_{s} \\
-\frac{L_{n d}}{\sigma L_{s d} L_{n d}} & -\omega_{s} & -\frac{R s L_{n d}^{2}+R, L_{n d d}^{2}}{\sigma L_{s d} L_{n d}^{2}}
\end{array}\right]\left(\begin{array}{l}
\psi_{r}(t) \\
i_{s M}(t) \\
i_{s T}(t)
\end{array}\right)+\left[\begin{array}{cc}
0 \\
0 \\
\frac{1}{\sigma L_{s d}} & 0 \\
0 & \frac{1}{\sigma L_{s d}}
\end{array}\right]\left(\begin{array}{l}
u_{s M}(t) \\
u_{s T}(t)
\end{array}\right)
$$

In the motor system, because of the induction switching phenomenon, the influence of the hysteresis state on the system is uncertain. Within a certain range, the degree of influence depends on the size of each element of the corresponding coefficient matrices. Here, definition $\mu(\Theta)$ is the influence factor, which represents the measurement of the degree of influence of state lag on the system in the model, where $\Theta$ is the corresponding coefficient matrix. definition:

$$
\mu(\Theta)=\frac{1}{\|\Theta\|_{\infty}}
$$

The state equation of the induction motor is converted into a standard time-delay system equation, and the following equation is obtained:

$$
\dot{x}(t)=(1-\mu) A_{0} x(t)+\mu A_{1} x(t-\tau)+B u(t)
$$

Equation (5) is derived from the Newton-Leibniz formula:

$$
\dot{x}(t)-\mu A_{1} \int_{0}^{\frac{\tau}{2}} \dot{x}(t-h+\theta) d \theta=(1-\mu) A_{0} x(t)+\mu A_{1} x(t-h)+B u(t)
$$

For the treatment of the integral term in equation (6), apply the discrete method to obtain the neutral motor system equation of the induction motor.

$$
\dot{x}(t)-\mu \bullet \frac{h}{\Delta N} A_{1} \sum_{i=1}^{N} \dot{x}\left(t-h-\frac{(i-1) \bullet \tau}{2 \Delta N}\right)=(1-\mu) A_{0} x(t)+\mu A_{1} x(t-h)+B u(t)
$$

Where $\Delta N \geq 1, \Delta N=1,2, \cdots N, h=\frac{\tau}{2}$.

\subsection{Design of neutral current controller and stability proof}

The equation of neutral system is as follows

$$
\left\{\begin{array}{l}
\dot{x}(t)-G \dot{x}(t-h)=A x(t)+A_{1} x(t-d)+B u(t) \\
x(t)=\phi(t), \quad t \in[-\max \{h, d\}, 0] \\
y(t)=C x(t)
\end{array}\right.
$$

Firstly, assume that the state of the system is observable, and design the following state observer :

$$
\hat{\dot{x}}(t)-G \hat{\dot{x}}(t-h)=A_{0} \hat{x}(t)+A_{1} \hat{x}(t-d)+L(y(t)-C \hat{x}(t))
$$

This makes the equation of error dynamic system (15) asymptotically stable.

$$
\dot{e}(t)-G \dot{e}(t-h)=A_{L} e(t)+A_{1} e(t-d)
$$

Where $A_{L}=A_{0}-L C ; e(t)$ is the error vector, $e(t)=x(t)-\hat{x}(t), \hat{x}(t) \in R^{n}$ is the observer state, and $L$ is the $n \times q$ order observer gain matrix.

Secondly, the linear state feedback controller is designed.

$$
U(t)=K x(t)
$$

Get the closed-loop system expression:

$$
\dot{x}(t)-G \dot{x}(t-h)=\left(A_{0}+B K\right) x(t)+A_{1} x(t-d)
$$

In order to prove the stability of the system equation (11), a Lyapunov functional is designed here. Its expression is as follows:

$$
\begin{aligned}
V\left(t, x_{t}, e_{t}\right) & =\left[\begin{array}{l}
x_{1}(t)-G x_{1}(t-h) \\
e_{1}(t)-G e_{1}(t-h)
\end{array}\right]^{T}\left[\begin{array}{cc}
P_{x} & 0 \\
0 & P_{e}
\end{array}\right]\left[\begin{array}{l}
x_{1}(t)-G x_{1}(t-h) \\
e_{1}(t)-G e_{1}(t-h)
\end{array}\right]+ \\
& \left.\int_{t-h}^{t}\left[\begin{array}{l}
x_{1}(\theta) \\
e_{1}(\theta)
\end{array}\right]^{T}\left[\begin{array}{cc}
Q_{x} & 0 \\
0 & Q_{e}
\end{array}\right]\left[\begin{array}{l}
x_{1}(\theta) \\
e_{1}(\theta)
\end{array}\right] \mathrm{d} \theta+\int_{t-d}^{t}\left[\begin{array}{c}
x_{1}(\theta) \\
e_{1}(\theta)
\end{array}\right]\right]^{T}\left[\begin{array}{cc}
R_{x} & 0 \\
0 & R_{e}
\end{array}\right]\left[\begin{array}{l}
x_{1}(\theta) \\
e_{1}(\theta)
\end{array}\right] \mathrm{d} \theta
\end{aligned}
$$

Where $P_{x}, P_{e}, Q_{x}, Q_{e}, R_{x}, R_{e}$ are the positive definite matrices to be determined. 
$\varepsilon=\left[\begin{array}{l}x_{1}(t)-G^{T} x_{1}(t-h) \\ e_{1}(t)-G^{T} e_{1}(t-h)\end{array}\right], P=\left[\begin{array}{cc}P_{x} & 0 \\ 0 & P_{e}\end{array}\right], Q=\left[\begin{array}{cc}Q_{x} & 0 \\ 0 & Q_{e}\end{array}\right], R=\left[\begin{array}{cc}R_{x} & 0 \\ 0 & R_{e}\end{array}\right]$ $\xi(t)=\left[\begin{array}{l}x_{1}(t) \\ e_{1}(t)\end{array}\right], \xi(t)=\left[\begin{array}{l}x_{1}(t) \\ e_{1}(t)\end{array}\right], \xi(t-h)=\left[\begin{array}{l}x_{1}(t-h) \\ e_{1}(t-h)\end{array}\right], J=\left[\begin{array}{cc}G^{T} & 0 \\ 0 & G^{T}\end{array}\right]$ $\rho(t-d)=\left[\begin{array}{l}x_{1}(t-d) \\ e_{1}(t-d)\end{array}\right]$

So the Lyapunov functional is written as:

$$
V(t, \xi(t))=\varepsilon^{T} P \varepsilon+\int_{t-h}^{t} \xi(\theta)^{T} Q \xi(\theta) \mathrm{d} \theta+\int_{t-d}^{t} \xi(\theta)^{T} R \xi(\theta) \mathrm{d} \theta
$$

Derivative along the solution of the closed-loop system equation (13), we have

$$
\begin{aligned}
\dot{V}(t, \xi(t))= & \varepsilon^{T}\left(P A_{0}+A_{0}^{T} P+Q+R\right) \varepsilon+2 \varepsilon^{T}\left(Q+R+P A_{0}\right) J \xi(t-h) \\
& +2 \varepsilon^{T} P A_{1} \xi(t-d)-\xi(t-h)^{T} \lambda \xi(t-h)-\xi(t-d)^{T} R \xi(t-d)
\end{aligned}
$$

Where $\lambda=Q-J^{T}(Q+R) J$

Since $P, Q, R$ are the positive definite matrices, $\dot{V}(t, \xi(t))$ can be equivalent to the expression (15).

$$
\begin{aligned}
\dot{V}(t, \xi(t))= & \varepsilon^{T}\left[P A_{0}+A_{0}^{T} P+Q+R+\left(Q+R+P A_{0}\right) J \lambda^{-1} J^{T}\left(Q+R+P A_{0}\right)^{T}\right. \\
& \left.+P A_{1} R^{-1} A_{1}^{T} P\right] \varepsilon-\left[\lambda \xi(t-h)^{T}-\varepsilon^{T}\left(Q+R+P A_{0}\right) J\right][\xi(t-h) \\
& \left.-\lambda^{-1} J^{T}\left(Q+R+P A_{0}\right)^{T} \varepsilon\right]-\left[R \xi(t-d)^{T}-\varepsilon^{T} P A_{1} R^{-1}\right][\xi(t-d) \\
& \left.-R^{-1} A_{1}^{T} P \varepsilon\right]
\end{aligned}
$$

Again by system equation (11),we have

$$
\begin{aligned}
\dot{V}(t, \xi(t)) \leq & \varepsilon^{T}\left[P A_{0}+A_{0}^{T} P+Q+R+\left(Q+R+P A_{0}\right) J \lambda^{-1} J^{T}(Q\right. \\
& \left.\left.+R+P A_{0}\right)^{T}+P A_{1} R^{-1} A_{1}^{T} P\right] \varepsilon
\end{aligned}
$$

Therefore, if there is a positive definite matrix $P, Q, R$, the inequality group (17) is established.

$$
\left\{\begin{array}{l}
P A_{0}+A_{0}^{T} P+Q+R+\left(Q+R+P A_{0}\right) J \lambda^{-1} J^{T}\left(Q+R+P A_{0}\right)^{T}+P A_{1} R^{-1} A_{1}^{T} P<0 \\
\lambda=Q-J^{T}(Q+R) J>0
\end{array}\right.
$$

Explain the existence of scalar $\gamma<0$, so that the following formula is established.

$$
\dot{V}(t, \xi(t)) \leq \gamma\|\varepsilon\|
$$

According to Razumikhin's type theorem [8], equation (12) is asymptotically stable, and the necessary and sufficient condition for the asymptotic stability of equation (12) is the asymptotic stability of system equation (11).

Therefore, if there are positive definite matrices $P_{x}, Q_{x}, R_{x}$ such that the inequality group is established, the closed-loop system equation (11) is uniformly asymptotically stable. Again by Schur complements the inequality group is equivalent to the matrix inequality (LMI).

$$
\left[\begin{array}{cccc}
P_{x}(A+B K)^{T}+(A+B K) P_{x} & P_{x}(A+B K)^{T} G^{T} & P_{x} A_{1}^{T} & I \\
G(A+B K) P_{x} & -Q_{x} & 0 & G \\
A_{1} P_{x} & 0 & -R_{x} & 0 \\
I & G & 0 & -\left(Q_{x}+R_{x}\right)^{-1}
\end{array}\right]<0
$$

Where $\mathrm{Z}=K P_{x}, F=A P_{x}+B \mathrm{Z}$, then we get $K=\mathrm{Z} P_{x}^{-1}$. Therefore, if there are positive definite matrices $P_{x} 、 Q_{x} 、 R_{x}$ that satisfies the above matrix inequality, and there must exist a state feedback controller $U=\mathrm{ZP}_{x}^{-1} x(t)$ to stabilize the system equation (11).

The summary design scheme of controller is as follows:
Step 1: solve the linear matrix inequality (18) to obtain positive definite matrices $P_{x} 、 Q_{x} 、 R_{x}$ and matrix Z;

Step 2: if there is a solution, $K=\mathrm{Z} P_{x}^{-1}$ is the gain matrix of feedback controller; if there is no solution, the positive definite matrices $P_{x} 、 Q_{x} 、 R_{x}$ and the matrix $\mathrm{Z}$ need to be solved again.

\section{Simulation results}

\subsection{Simulation parameters of induction motor}

Table 1 shows the simulation parameters of induction motor .

Table 1. Motor parameters

\begin{tabular}{cc}
\hline Parameter & Value \\
\hline Power rating $/ \mathrm{kW}$ & 4 \\
Rated frequency $/ \mathrm{Hz}$ & 50 \\
Rated voltage $/ \mathrm{V}$ & 380 \\
Rated cuerent $/ \mathrm{A}$ & 8.7 \\
stator resistance $/ \Omega$ & 1.405 \\
rotor resistance $/ \Omega$ & 1.395 \\
Rotor leakage $/ \mathrm{mH}$ & 5.839 \\
Sampling time $/ \mathrm{ms}$ & 2 \\
Moment of inertia $J / \mathrm{kg} \cdot \mathrm{m}^{2}$ & 0.511 \\
Rotor mutual inductance $/ \mathrm{mH}$ & 172.2 \\
Polar logarithm & 2 \\
\hline
\end{tabular}

The above parameters are introduced into the neutral time delay system model of induction motor, and the coefficient matrices of the system equation are obtained.

$$
\begin{aligned}
G & =\left[\begin{array}{ccc}
-0.0018 & 0.0003 & 0 \\
0.1497 & -0.0535 & -2.253 \omega_{s} e-04 \\
-0.9998 & -2.253 \omega_{s} e-04 & -0.0535
\end{array}\right] \\
A_{0} & =\left[\begin{array}{ccc}
-7.8 & 1.3 & 0 \\
664.3 & -237.5 & \omega_{s} \\
-4435.8 & -\omega_{s} & -237.5
\end{array}\right] \\
A_{1} & =\left[\begin{array}{ccc}
-0.0018 & 0.0003 & 0 \\
0.1497 & -0.0535 & -2.253 \omega_{s} e-04 \\
-0.9998 & -2.253 \omega_{s} e-04 & -0.0535
\end{array}\right] \\
B & =\left[\begin{array}{cc}
0 & \\
87.6347 &
\end{array}\right]
\end{aligned}
$$

Where $\omega_{s}$ is an induction motor stator rotating magnetic field synchronous angular velocity. In the simulation, according to the real-time speed calculation of motor, it is a time-varying parameter. The speed of the motor is $n=500 \mathrm{r} / \mathrm{min}$. When the switching frequency is $500 \mathrm{~Hz}$, and the system delay time [1] is $h=1.5 T_{s}=3 \mathrm{~ms}$.

First use the feasp function of LMI toolbox in Matlab to solve the linear matrix inequality group (17), and obtain the positive definite matrices $P_{x} 、 Q_{x} 、 R_{x}$ and the matrix $\mathrm{Z}$. Then you can find the gain matrix $K$ of feedback controller, as follows: 


$$
\begin{aligned}
& P_{x}=\left[\begin{array}{ccc}
0.006 & -0.111 & -1.101 \\
-0.111 & 1.007 & 1.754 \\
-1.101 & 1.754 & 3.014
\end{array}\right], Q_{x}=\left[\begin{array}{ccc}
0.501 & -0.057 & -0.126 \\
-0.057 & 6.520 & -0.612 \\
-0.126 & -0.612 & 10.564
\end{array}\right] \\
& R_{x}=\left[\begin{array}{ccc}
0.341 & -0.049 & -0.117 \\
-0.049 & 5.597 & 0.013 \\
-0.117 & 0.013 & 5.219
\end{array}\right] \\
& \mathrm{Z}=\operatorname{diag}\{-0.310,3.419,-7.667\} \\
& K=\operatorname{diag}\left\{a_{1}, a_{2}, a_{3}\right\}=\operatorname{diag}\{-48.310,3.395,-2.544\}
\end{aligned}
$$

The above positive definite matrices and $a_{1}, a_{2}, a_{3}$ are dynamically changing. A set of solutions for stabilizing the motor speed is given here, which proves that neutral time-delay systems are stable. Finally, the relationship between the control input $U_{s}(t)$ and the controlled object $i_{s}(t)$ is obtained by the theory of neutral delay system, and the relationship between the two phase stator voltage and the two phase stator current is obtained. The gain matrix $K$ of feedback controller is the neutral current controller designed in this paper.

$$
\left\{\begin{array}{l}
u_{s M}(t)=a_{2} \cdot i_{s M}(t) \\
u_{s T}(t)=a_{3} \cdot i_{s T}(t)
\end{array}\right.
$$

\subsection{Simulation of neutral current controller}

In this paper, Matlab/Simulink/S-Function programming is used to simulate the operating mode of the microprocessor to the maximum, and a simulation model of the induction motor based on the neutral current controller is built to verify the neutral current controller. Figure 1 is schematic diagram of simulation model of induction motor based on neutral current controller. The NDS-ACR is the neutral current controller.

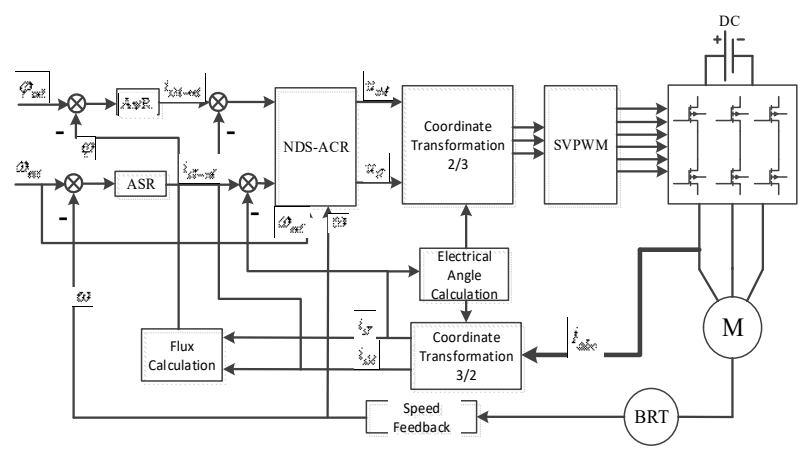

Fig. 1. Schematic diagram of simulation model of induction motor based on neutral current controller.

The given speed in the simulation is $500 \mathrm{r} / \mathrm{min}$. Figure 2 shows the speed response of a neutral current controller. From Figure 2, it can be seen that the speed adjustment time is $0.1 \mathrm{~s}$, which shows the response speed of motor with the neutral current controller is Very fast, the adjustment range of adjustment time is larger than the traditional methods.

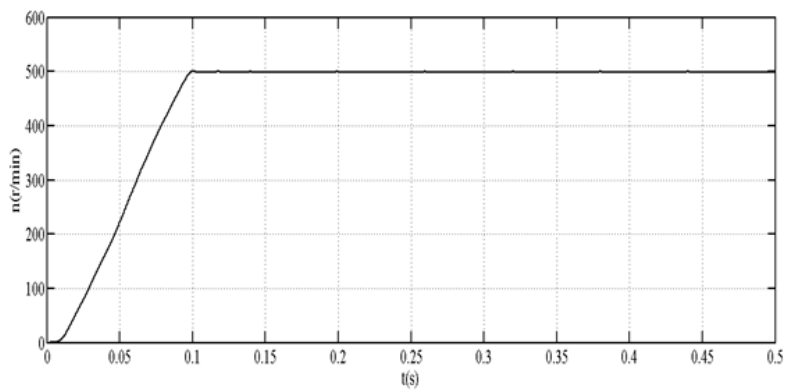

Fig. 2. Rotation speed response diagram of neutral method.

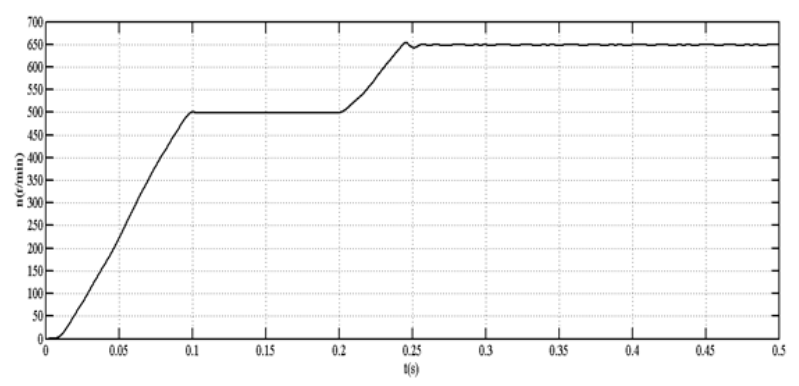

Fig. 3. The speed response diagram of neutral method.

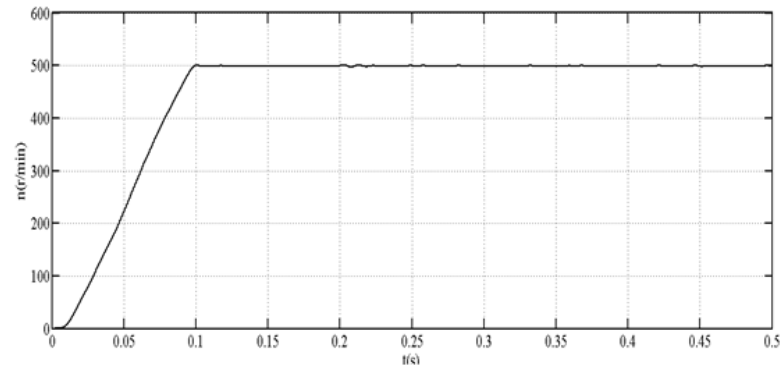

Fig. 4. Loaded speed response diagram of neutral method.

After the motor speed reaches the given speed of 500 $\mathrm{r} / \mathrm{min}$, the speed is adjusted to $650 \mathrm{r} / \mathrm{min}$ at $0.2 \mathrm{~s}$. Figure 3 is a simulated waveform diagram of the motor speed. After the motor speed reaches $500 \mathrm{r} / \mathrm{min}$, add load $50 \mathrm{~N} \cdot \mathrm{m}$ at $0.2 \mathrm{~s}$. Figure 4 is a simulated waveform of the motor speed after loading.

As can be seen from Figure 3, when the neutral current controller is used, the speed regulation waveform of the motor is smooth, and the speed response is fast. In less than $0.05 \mathrm{~s}$, the speed is stabilized again. The regulation performance of speed is good. From Figure 4, we can see that, adds load after the speed is stabilized, and the fluctuation of instantaneous rotation speed is lower than $\pm 4 \mathrm{r} / \mathrm{min}$. The rate of rotation speed fluctuation is less than $\pm 0.8 \%$, indicating that the rotation speed control of motor has strong anti-interference ability and strong robustness. 


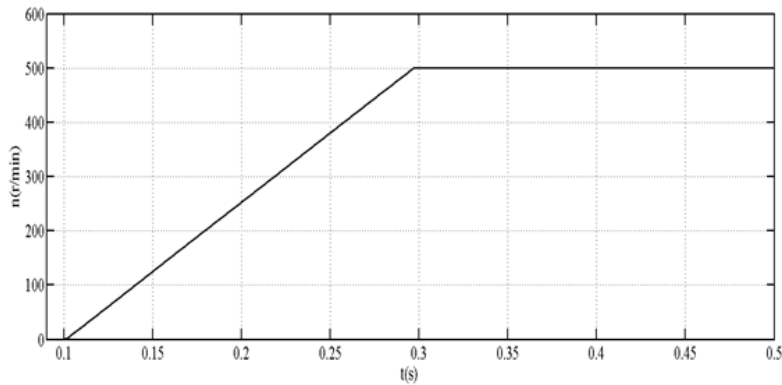

Fig. 5. Speed chart of neutral method.

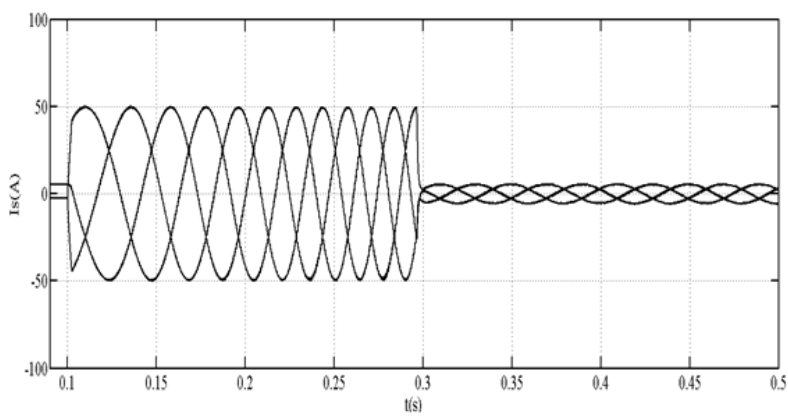

Fig. 6. The stator current waveform of neutral method.

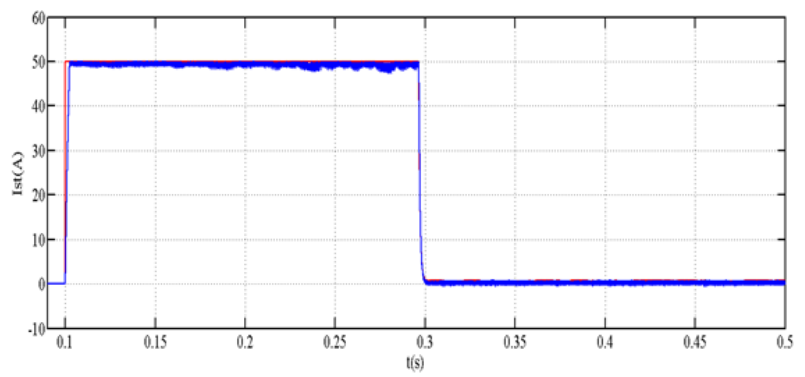

Fig. 7. Current torque component following curve of neutral method.

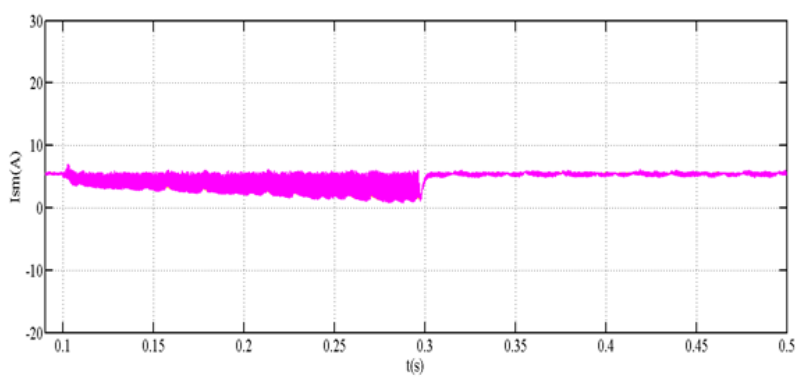

Fig. 8. Current excitation component following curve of neutral method.

At a given base speed of $500 \mathrm{r} / \mathrm{min}$, the starting speed is raised from 0 to $500 \mathrm{r} / \mathrm{min}$ at $0.1 \mathrm{~s}$. Figures $5,6,7$ and 8 are simulation results of the neutral current controller in the M-T coordinate system. Figure 5 shows the speed curve. Figure 6 shows the stator current curve of induction motor. Figure 7 shows the stator current torque component following curve. Figure 8 shows the stator current excitation component follow curve.

It can be seen from Figure 5 and 6 that in the process of speed increase and abrupt change, the speed response is rapid, the stator current curve is smooth, and the current fluctuation is small, the steady-state error is almost 0 , and the robustness is strong. From Figure 7 and 8 , it can be seen that the stator current component of the neutral current decoupling control method has very little fluctuation, the fluctuation rate of the current torque component is lower than $3 \%$, the fluctuation of current excitation component is lower than $4 \mathrm{~A}$. The actual stator current torque component and the excitation components of induction motors can quickly follow the given values of the respective stator current component, and the decoupling effect of the current is significant. The above results show that using a neutral current controller can make induction motors have good steady-state and dynamic performance.

Compared with the traditional method, the neutral controller has significantly improved the dynamic response of the motor, the speed response time of motor is short, and the speed regulation is fast. The induction motors have strong anti-interference ability. The impact of the digital delay on the system performance is considered in the simulation. The neutral type is adopted, the digital delay of the controller has little effect on the system.

\section{Conclusion}

In this paper, the traditional control methods of induction motors cannot solve the problem of digital delay on the system, and propose a neutral-based current control method for induction motor to design a neutral current controller. The simulation results show that:

a. Neutral current controller NDS-ACR is realized through software programming, and the parameters are automatically set, avoiding trial and error, high efficiency and good timeliness.

b. Neutral math modeling of induction Motor contains digital delay terms so that makes the model more accurate. The system using a neutral current controller has the advantages of a large range of speed adjustment time, fast system response, and strong robustness.

c. Speed control system of induction motors using neutral current control method, two-phase stator current cross-coupling weakened, decoupling effect is obvious. The fluctuation of stator current torque component and excitation component is small. The control effect is good, and has a good application prospects.

The authors acknowledge the National Natural Science Foundation of China (51331002) and the Open project of State Key Laboratory of Power System of Tsinghua University (SKLD16KZ07).

\section{References}


1. L.Y. Qi, T.T. Wang, M.L Zhou, J Wang. $A$ Decoupling Current Control Scheme for Induction Machine Controllers. Trans of China Elec Society, 29, 174-180(2014).

2. J. Holtz, J.T. Quan, et al. Design of fast and robust current regulators for high-power drives based on complex state variables. Trans on Industry Appl, 40, 1388-1397(2004).

3. K.K. Wei, M.L. Zhou, Q.L. Zheng, et al. Discrete-time current controller for induction motors based on complex vector. Trans of China Elec Society, 26, 88-93(2011).

4. E. Monmasson, M.N. Cirstea. FPGA design methodology for industrial control systems-a review. IEEE Trans on Industrial Electronics, 54, 1824-1842(2007).
5. G.H. Bode, P.C. Loh, et al. An improved robust predictive current regulation algorithm. IEEE Trans on Industry Appl, 41, 1720-1733(2005).

6. Z. Wang, J. Lam, and K.J. Burnham. Stability analysis and observer design for neutral delay systems. IEEE Trans on Auto Control, 47, 1321-1326(2001).

7. W.j. Li, Y Ruan, H.Q. Gu. Decoupling of current and design of controller for vector control system. $\mathrm{J}$ of motor and control appl, 35, 18-22(2008).

8. J. H. Park and R. Won. Stability analysis for neutral delay differential systems. J of Franklin Institute, 337, 1-9(2000). 